\title{
Entornos virtuales en la formación docente como mediación de enseñanza de las I.E. de cuarto grado de primaria de la Ugel Norte
}

Virtual environments in teaching training as a teaching mediation of the I.E. of fourth grade of primary of the North Ugel

Silvia Dolores Quispe Flores ${ }^{1}$, Arabella Bridget Mena Alarcón ${ }^{1}$ y Lita Marianela Quispe Flores².

${ }^{1}$ Instituto Superior Pedagógico, Arequipa, Perú.

${ }^{2}$ Universidad Nacional San Agustin, Arequipa, Perú.

\section{INFORMACIÓN}

\section{Historia del Artículo}

Recepción: 15/04/2019

Revisión: $12 / 07 / 2019$

Aceptación: 20/08/2019

\section{Palabras Clave}

Redes de comunicación, medios tecnológicos, entornos virtuales de aprendizaje, herramientas digitales.

\section{Key Words}

Communication networks, technological means, virtual learning environments, digital tools

\section{DOI}

https://doi.org/10.35286/veritas. v21i1.253

\section{RESUMEN}

La presente investigación se analizó, de los resultados obtenidos los entre los años 2007 2014 aplicados por el Sistema Regional de Evaluación de los Aprendizajes (SIREVA), cuyos resultados nos mostró la brecha existente de niños que no alcanzaron un nivel satisfactorio en comprensión lectora y en matemática, para realizar el estudio de datos se utilizó la base de datos de alcance regional correspondiente a la UGEL Norte, a partir de esta información obtenida se analizó material bibliográfico sobre entornos virtuales que den soporte a la mejora del proceso enseñanza aprendizaje en los docentes, llegamos a evidenciar los efectos positivos del uso de herramientas digitales en la aplicación del programa. En los ultimos cinco años se ha venido trabajando un conjunto de estrategias a fin de mejorar los niveles de comprension lectora y matematica en los estudiantes de Segundo y cuarto grado de primaria en la instituciones educativas de la ugel Arequipa norte sin embargo aun se encuentran en un $40 \%$ en el nivel de proceso, no logrando las capacidades y desempeños minimos hacia el logros delas competencias del area de matematica y comunicacion, siendo el problema el uso de estrategias de enseñamza y aprendizaje pertinenete para lograr las competencias en un nivel satisfactorio, es asi que uno de los medios para logra la efectividad de la estrategia es el uso de los entornos virtuales. objetivo general fortalecer la formación docente de la mediación de la enseñanza con el uso de los entornos virtuales a través de la implementación del proyecto "Mejoramiento de la calidad del servicio educativo para incrementar el logro de aprendizajes en comunicación y matemática en estudiantes de Instituciones Educativas Públicas del nivel primario, correspondientes al ámbito de intervención de la UGEL Norte" y tiene como objetivos específicos: a) Analizar el manejo de los entornos virtuales como herramientas de medición de la enseñanza, b) Analizar los factores que influyen mejoramiento de su formación profesional con el uso de entornos virtuales. Selección de los participantes docentes que intervinieron en las siguientes etapas del proyecto "Mejoramiento de la calidad del servicio educativo para incrementar el logro de aprendizajes en comunicación y matemática en estudiantes de Instituciones Educativas Públicas del nivel primario, correspondientes al ámbito de intervención de la UGEL Norte. Se realiza las siguientes intervenciones: 1 Uso de la plataforma, 2 Uso del software educativo, 3 Uso de los videos en las pasantías docente, 4 Capacitaciones en la planificación curricular y el manejo de entorno virtuales, 5 Monitoreo y Acompañamiento. Muestra: Se seleccionó una muestra al azar de todas las IE de la u gel norte de educación primaria, teniendo una muestra de 84 IE que corresponde a 84 docentes 10 directores En cuanto a los resultados En las respuestas de los 82 docentes y directores encuestados 34\%(28 de los 82 evaluados) de ellos afirmo que las estrategias pedagógicas han permitido mejorar los aprendizajes de sus estudiantes. 


\begin{abstract}
effectiveness of the strategy is the use of virtual environments. general objective to strengthen the teaching training of the mediation of education with the use of virtual environments through the implementation of the project "Improving the quality of the educational service to increase the achievement of learning in communication and mathematics in students of Public Educational Institutions of the primary level, corresponding to the scope of intervention of the UGEL North "and has as specific objectives: a) Analyze the management of virtual environments as teaching measurement tools, b) Analyze the factors that influence the improvement of their professional training with the use of virtual environments. Selection of the teaching participants who participated in the following stages of the project "Improving the quality of the educational service to increase the achievement of learning in communication and mathematics in students of Public Educational Institutions of the primary level, corresponding to the scope of intervention of the North UGEL. The following interventions are performed: 1 Platform use, 2 Use of educational software, 3 Use of videos in teacher internships, 4 Training in curriculum planning and virtual environment management, 5 Monitoring and Accompaniment, Sample: A random sample of all the EIs of the northern primary education gel was selected, with a sample of 84 EI corresponding to 84 teachers and 10 directors As for the results In the answers of the 82 teachers and principals surveyed, $34 \%$ ( 28 of the 82 evaluated) said that the pedagogical strategies have improved the learning of their students
\end{abstract}

\section{INTRODUCCIÓN}

En el año 2009, a nivel de la Región Arequipa surge una iniciativa importante como es el Sistema Regional de Evaluación de los Aprendizajes (SIREVA), con el objetivo de obtener información de las II.EE en las áreas de ciudadanía, matemática y comunicación de primero a sexto de primaria, a través de la aplicación de pruebas de entrada, proceso y salida para que la comunidad educativa pueda tomar decisiones en base a:

- I. Los diferentes niveles de logro de las competencias por cada estudiante;

- II. Las capacidades críticas (diagnóstica, pre requisito y capacidades seleccionadas del grado) de las áreas curriculares de comunicación y matemática;

- III. El nivel de eficacia en el avance del proceso pedagógico;

- IV. El avance de los niveles de logro de las competencias a nivel del aula y grado.

Desde el año 2013 este sistema está extendido en el 100\% de II.EE públicas de la Región Arequipa y se ha constituido en un instrumento de manejo interno; que permite a las II.EE generar alternativas pedagógicas que van atendiendo la particularidad de la II.EE, sin embargo, al no contar con recursos adicionales la información no es procesada a nivel regional, de tal forma que su contribución a la formación de un modelo pedagógico regional; está aún siendo postergado.

La lectura de los principales antecedentes e hitos relevantes vinculados al logro de aprendizajes detallados en los párrafos anteriores, nos habla de una serie de intervenciones, muchas de ellas dispersas y aisladas, que no necesariamente han conversado en la parte operativa a pesar de buscar el mismo objetivo.

Por otro lado, respecto a los resultados de las evaluaciones censales, no necesariamente ha tenido la misma dinámica que el incremento del presupuesto; aun cuando han mejorado de manera significativa entre el 2013 y 2014, como se muestra en el gráfico $\mathrm{N}^{\circ} 1$. Aún existe una brecha del $39 \%$ de niños que no alcanzan un nivel satisfactorio en comprensión lectora y un $67 \%$ en matemáticas, parte de esta población está en las II.EE públicas poli docente de la UGEL Norte, donde la intervención de acompañamiento pedagógico es muy reducida.

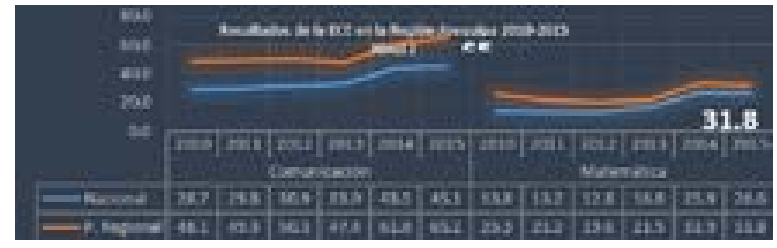

Fig. 1: Resultados de la evaluacion censal 2007-2014 en base a SICRECE. Fuente: Elaboracion propia

El Gobierno Regional de Arequipa en línea con las políticas educativas nacionales, regionales y considerando las prioridades establecidas en su plan de gobierno 20152018, realiza un balance de estas intervenciones educativas y los resultados obtenidos desde el 2007 hasta fecha en las evaluaciones de los aprendizajes y decide impulsar la formulación de un estudio de pre inversión que permita reducir las brechas existentes en la Región, haciendo énfasis en una intervención integral que aborde factores clave y de probada evidencia asociados al aprendizaje, como el desempeño docente y directivo, la gestión territorial educativa concertada y el monitoreo y evaluación por competencias, involucrando a los diferentes actores e instituciones vinculadas al servicio educativo.

El propósito del Gobierno Regional es atender a todas las II.EE públicas que actualmente no están priorizadas en los Programas Nacionales del MINEDU; en ese sentido propone una intervención integral en II.EE públicas poli docente de la UGEL NORTE, que cobertura el $26 \%$ de la población escolar del nivel primario en la Región Arequipa y el acompañamiento pedagógico del PELA está en el 2.8\% de las escuelas. Así mismo, los resultados de la evaluación de los aprendizajes en comunicación y matemáticas en la UGEL NORTE, han mostrado en los últimos 4 años (20102014) una curva de crecimiento irregular de incrementos poco significativos y descensos hasta el 2013 y un salto significativo en el 2014; aun así, hay una brecha importante que atender como se muestra en la tabla 1. 
VÉRITAS Vol. 21 N¹ (2020) 13-24

Tabla 1: Resultado de la Evaluación Censal 2007-2014. Fuente: UMC -MED

\begin{tabular}{ccccccccc}
\hline ÁREAS & 2007 & 2008 & 2009 & 2010 & 2011 & 2012 & 2013 & 2014 \\
\hline $\begin{array}{c}\text { Comprensión } \\
\text { Lecto-ra }\end{array}$ & 41.5 & 42.3 & 46.8 & 55.0 & 55.7 & 58.2 & 53.2 & 65.7 \\
Matemática & 14.6 & 17.6 & 29.2 & 29.4 & 24.7 & 26.0 & 25.5 & 37.9 \\
\hline
\end{tabular}

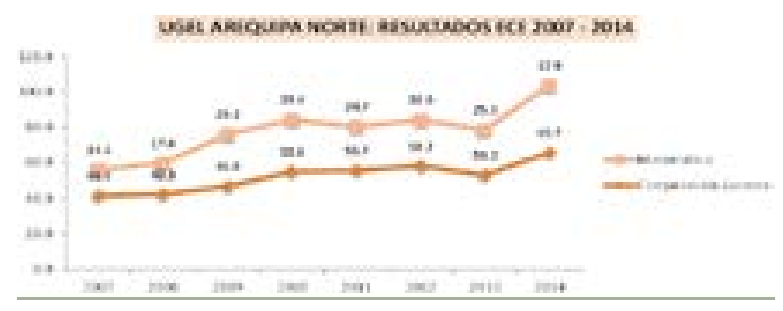

Fig. 2: Resultados de la evaluación censal 2007-2014.

La lectura de los resultados de la evaluación censal 2007-2014; a nivel nacional, regional y local nos lleva a la conclusión, que existe una brecha importante por la cual es necesario continuar haciendo esfuerzos articulados para mejorar los aprendizajes de los estudiantes.

Es importante mencionar que el proyecto se encuentra en el marco de los lineamientos de la política educativa actual, a nivel nacional, regional y local. Esta pertinencia ha sido analizada y se presenta en la siguiente tabla 2 :

Tabla 2: Política actual a nivel nacional, regional y local
Por lo cual una de los factores que influyen en lograr estos objetivos orientado a la calidad educativa es el docente desde su formación docente que como mediador se debe de apoyar en los recursos virtuales para mejorar su proceso de enseñanza y aprendizaje.

Es así que en la actualidad una de las mayores aportaciones de las nuevas tecnologías de la información y las comunicaciones (TIC) a la educación son sin duda los Entornos Virtuales de Formación (EVF), también llamados Entornos Virtuales de Enseñanza-Aprendizaje (EVEA) o Sistemas de Tele formación (STF) [6]

Según [7] hay diferentes aspectos que justifican la necesidad de formación y perfeccionamiento del profesorado en TIC, entre ellos destaca fundamentalmente tres:

- Los nuevos contextos, espacios de la sociedad de la información.

- Las características de los nuevos entornos formativos virtuales en el campo de la educación.

- Los nuevos roles y funciones que se le están asignando al profesorado en las sociedades e instituciones educativas del futuro

OBJETIVOS ESPECIFICOS DEL MILENIO [2]

Lograr la enseñanza primaria universal.

PROYECTO EDUCATIVO NACIONAL 2021[3]

Objetivo Estratégico 2:

Estudiantes e instituciones que logran aprendizajes pertinentes y de calidad.

Objetivo Estratégico 4. Una gestión descentralizada, democrática, que logra resultados y es financiada con equidad.

MARCO DEL BUEN DESEMPEÑO DOCENTE (Resolución Ministerial No. 0547-2012-ED)[4]

MARCO DEL BUEN DESEMPEÑO DIRECTIVO [5]
Política 7.1: Asegurar prácticas pedagógicas basadas en criterios de calidad y de respeto a los derechos de los niños.

Política 7.3: Fomentar climas institucionales amigables, integradores y estimulantes.

Política 8.2: Establecer programas de apoyo y acompañamiento pedagógico con funciones permanentes de servicio a las redes escolares. Política 15. Fortalecer una participación social responsable y de calidad en la formulación, gestión y vigilancia de las políticas y proyectos educativos.

El Marco de Buen Desempeño Docente de $\neg$ fine los dominios, las competencias y los desempeños que caracterizan una buena docencia y que son exigibles a todo docente de Educación Básica Regular del país. En ese sentido, establece cuatro dominios que comprenden nueve competencias y cuarenta desempeños.

El Marco de Buen Desempeño del Directivo define los dominios, competencias y desempeños con sus correspondientes descriptores los cuales caracterizan una dirección escolar eficaz que refuerza el liderazgo de los directivos de las instituciones de educación básica del país. En ese sentido establece dos dominios que comprenden seis competencias y 21 desempeños. competencias están en el Marco del Buen Desempeño
El proyecto tiene como objetivo, in-crementar el nivel de logro de apren-dizajes en comunicación y matemática del nivel primario de instituciones públicas, lo cual contribuye con este objetivo del milenio.

El proyecto en su primer componente, propone la asistencia técnica a docentes y directivos para que puedan manejar recursos y estrategias pedagógicas que les permita lograr aprendizajes significativos a los estudiantes, así como un clima adecuado en el aula y en la Institución Educativa, este componente está directamente vinculado con las políticas 7.1, 7.3 y 8.2 del Proyecto Educativo Nacional.

El proyecto en su segundo componente propone un gestión territorial concertada del servicio educativo través de la elaboración y ejecución el Proyecto Educativo Local, fortaleciendo de esta manera la participación social como lo menciona la política 15 del Proyecto Educativo Nacional.

El proyecto en su primer componente propone mejorar el desempeño docente a través del fortalecimiento de sus competencias en aspectos de programación curricular, aplicación de estrategias para el aprendizaje y convivencia democrática en el aula y para la evaluación de los estudiantes. Todas estas competencias están en el Marco del Buen Desempeño Docente.

El proyecto en su primer componente propone mejorar el desempeño directivo a través del fortalecimiento de sus competencias en aspectos de gestión pedagógica y gestión institucional que le permitan liderar los procesos pedagógicos y stablecer condiciones adecuadas en la Institución para el aprendizaje de los estudiantes. Todas estas Directivo. 
Nosotros añadiríamos un nuevo concepto para justificar la formación del profesorado, y es la imperiosa necesidad de cambiar las actitudes del profesor ante el uso de las TIC en su quehacer docente.

Necesidad que crece con la formación del Espacio Europeo de Educación Superior (EEES) ya que se genera una nueva forma de ver la enseñanza centrado en el aprendizaje del alumno y donde las TIC son herramientas imprescindibles en el mismo. La concepción de la enseñanza en este sentido provoca multitud de cambios educativos, podemos hablar de redes de comunicación y transmisión de información que facilitan la interactividad continua y permanente entre los estudiantes, eliminando las barreras espacio-temporales y sus condicionantes. Así pues, parece lógico que las instituciones educativas se planteen cómo utilizar mejor las TIC en la enseñanza. [8]

En ese contexto la intervención de los entornos virtuales como herramientas de enseñanza es importante ya que permite despertar la motivación, el interés, por aprender y así mejorar los logros de aprendizaje del área de comunicación y matemática.

Es necesario destacar que la demanda educativa internacional requiere que se inserte las TIC en el campo educativo de las Instituciones Educativas que permita tener la base para la educación universitaria. Aun siendo evidente las ventajas, las TIC no están siendo fáciles de introducir en la universidad, para ello se requiere de un esfuerzo de aprendizaje y motivación por parte del profesor. De hecho, en este nuevo entorno universitario ya no debería considerarse la docencia virtual como algo separado de lo presencial, puesto que en muchas ocasiones el docente tendrá que valerse de las TIC para llevar a cabo su trabajo. [9]

Es necesario también el esfuerzo y la motivación del docente de la educación básica regular más aun ya que el estilo y ritmo de aprendizaje de los estudiantes es más dinámico, activo y que debe ser abstracto con una representación mental más cerca de la realidad y lograr la visualización e interpretación de los fenómenos de la naturaleza y contexto.

Las IE con una formación docente que domine y utilice los entornos virtuales debe los directores gestionar las condiciones de gestión curricular como el tiempo, espacio, recursos virtuales, capacitación de los entornos virtuales, motivación, e incluso cambiando su cultura organización, como reestructurar los horarios, las modalidades de enseñanza presencial y virtual, retroalimentación virtual, seguimiento y nivelación. De igual forma los docentes deben de formar círculos de interaprendizaje, pasantías, tertulias virtuales, capacitación en los entornos virtuales que les permita consolidad su formación docente, que le permita cortar las brechas entre la educación básica regular y la educación superior universitaria ya que, al mismo tiempo, deben asumirse una serie de cambios en las estructuras universitarias actuales que precisan, en muchos casos, de una ayuda externa a las organizaciones. Apostando por la necesidad de facilitar el aprendizaje y la docencia virtual desde la Institución universitaria, promoviendo la flexibilidad del lugar de docencia y del horario, la adaptación al ritmo de aprendizaje del alumno, llevando a cabo un aprendizaje no lineal e interactividad entre agentes docentes, para facilitar al docente la realización de todas sus tareas, entre las que se encuentra la gestión de los aprendizajes de los alumnos, y la mejora adaptación al cambio que se avecina.[9]

\section{La Sociedad Del Conocimiento Como Nuevo Escenario De La Formación}

Nadie pone en duda, independientemente de lo acertado, o no, del término "Sociedad de la Información y del Conocimiento", que hemos pasado a un modelo de sociedad notablemente diferente al existente a finales del siglo XX. A grandes rasgos la sociedad ha pasado por diferentes estadios de evolución: agrícola, industrial, postindustrial y de la información.

A lo largo de la historia, la disponibilidad de los nuevos medios tecnológicos ha actuado como una nueva puerta que se abre hacia un mundo de posibilidades. De repente, lo que hasta entonces era impensable se hace posible. Las tecnologías se materializan en nuevas infraestructuras, es decir, en nuevas herramientas que permiten la ejecución de los procesos de una forma más conveniente. [10]

Ante esta sociedad de conocimiento los docentes deben estar a la par de estas exigencias y además considerar que por muchos años se incidió en las capacitaciones direccionadas por el ministerio de educación que muchas veces no satisface las expectativas del docente en función a su realidad educativa y del aula es por eso que en la actualidad funciona como proceso de capacitación los temas de las TIC, pasantías, análisis de videos, uso de las plataformas virtual etc.

Así, una forma de clasificar la evolución de la sociedad es tomar como referencia la tecnología dominante de codificación, almacenamiento y recuperación de información [11]Se piensa que las condiciones sociales, políticas, económicas y culturales que caracterizan a las sociedades del siglo XXI han permitido, entre otras cosas, el surgimiento de lo que se conoce como la cultura de la sociedad digital [12]

Se menciona que, en una sociedad así caracterizada, las tecnologías digitales aparecen como las formas dominantes para comunicarse, compartir información y conocimiento, investigar, producir, organizarse y administrar. En este contexto, se reflexiona sobre la capacidad transformadora que las tecnologías de la información y la comunicación (TIC) representan para la educación en la denominada "sociedad del aprendizaje", "sociedad del conocimiento" o "sociedad-red". [12]

\section{La formación del profesorado en la Sociedad del conocimiento}

La tarea docente es una de las que más necesitan ser repensadas en el contexto de la sociedad del conocimiento y más concretamente la del docente. La utilización de las TIC permite llevar a la práctica nuevas metodologías que nos ayudan a adaptar el entorno educativo a las necesidades que la sociedad demanda.

"La sociedad del conocimiento y el nuevo EEES imponen y exigen nuevas competencias al desarrollo profesional y a la práctica educativa de la Universidad Española y de las IE, que, aunque nunca deberá perder su esencia, tampoco puede quedarse impasible ante el proceso de innovación de casi todo lo que nos rodea" [13]

La incorporación de las TIC en las universidades puede suponer un salto cualitativo de las mismas y como suele ocurrir cada vez que un nuevo desafío llama a las puertas de nuestras instituciones educativas, el profesorado es considerado como la clave del éxito del mismo. La interrogación acerca de las competencias que posee y las que no, la utilización que ya 
está haciendo, las necesidades de formación derivadas, los apoyos disponibles, los tiempos y espacios necesarios para las exigencias del cambio, etc., se convierten en motivos de preocupación e indagación para los investigadores de la enseñanza superior [14]

Para Margalef y Álvarez [15] la formación del profesorado en la sociedad del conocimiento debería tener unas premisas básicas:

El reconocimiento del saber experiencial de profesores y profesoras concretos, con historias de vida, trayectorias profesionales, con una determinada relación con el conocimiento en función de la epistemología de su campo científico, un estilo de enseñanza, una determinada situación laboral... que hablan de su diversidad ya que en la universidad no sólo los estudiantes son diversos, sino que también los profesores y profesoras lo son.

- Partir de la experiencia acumulada no significa sacralizarla y hacer de las rutinas criterios de actuación que refuerzan la resistencia al cambio, generan la tranquilidad de moverse en un terreno conocido frente a la incertidumbre y el desasosiego generados por lo "nuevo" y lo desconocido. Examinar esta creencia, trabajarla y, si procede, modificarla, supone un trabajo de reconstrucción y búsqueda de formas alternativas de realizar la enseñanza. Llevarlo a la práctica, exige contextos de formación que faciliten oportunidades para vivenciar un proceso de aprendizaje relevante.

- La reflexión sobre la práctica docente como potencial generador de pensamiento pedagógico, como fuente de innovación, en solitario o con otros. La transformación de la práctica pasa necesariamente por ese estadio, pero también por la necesidad de tiempos y apoyos para la formación. La sugerencia para ello de estrategias de trabajo cooperativo y en equipo.

- Buscar coherencia entre la teoría y la práctica, de manera que aquello que se considere valioso para la formación de nuestros estudiantes pueda ser experimentado por profesores y profesoras universitarios en sus procesos de formación. Una tarea exigente que pone de manifiesto la propia complejidad de la tarea de enseñar. Unas propuestas que contrastan con la socialización del profesorado universitario en su largo aprendizaje de la observación como alumnos.

\section{Características De Los Nuevos Entornos De Formación}

La situación generada por las TIC en las universidades y la educación básica, está influyendo con claridad para que se creen nuevos escenarios formativos, que, como comentábamos cuando hablábamos sobre los escenarios formativos de los EVF, rompen con la trilogía: tiempo, espacio y actividad.

Estos nuevos escenarios formativos plantean desafíos técnicos y pedagógicos a los que los profesionales deben responder. La desaparición del espacio físico de estas modalidades formativas crea de esta manera un mercado global en el que las instituciones educativas llamadas ya "tradicionales" compiten entre sí con nuevas iniciativas formativas tanto públicas como privadas. Además, estos nuevos escenarios vienen determinados por una serie de hechos significativos como son [7]:
- Que el conocimiento deja de ser lento, escaso y estable. Desde distintos lugares del mundo los hombres producen conocimientos y los difunden rápidamente con la ayuda de Internet.

- Que la educación formal ha dejado de ser el canal único mediante el cual las nuevas generaciones entran en contacto con el conocimiento y la información. Los medios de comunicación y las redes electrónicas se han transformado en grandes colaboradores, competidores o enemigos del educador, según sea la forma como se.

- Y que las instituciones educativas ya no pueden actuar más como si las competencias que forma, los aprendizajes a que da lugar y el tipo de inteligencia que supone en los alumnos, pudieran limitarse a expectativas formadas tradicionalmente. Las TIC y la apertura hacia la economía global basada en el conocimiento, obligan a desarrollar otros saberes y competencias para el abordaje de la esta nueva sociedad.

Las características que tienen estos nuevos escenarios formativos son: Tecnológicas y mediáticas, amigables, flexibles, individualizados, colaborativos, activos, interactivos/dinámicos, deslocalizados espacialmente de la información, pluripersonales, y pluridimensionales/ multiétnicos.

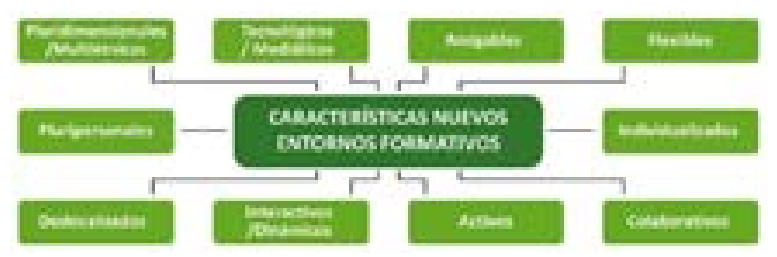

Fig. 3 Características de los nuevos entornos virtuales. Adaptado de [7]

\section{Planeación Educativa}

El alcance de la planeación hace necesario contemplar las dimensiones que se relacionan con ella, entre las que destacan:

Dimensión Social: Ya que la planeación es realizada por grupos humanos, no puede escapar de su carácter social pues son los propios individuos quienes se verán afectados con la implantación de algún plan, programa o proyecto.

Dimensión Técnica: Toda planeación supone el empleo de conocimientos organizados y sistemáticos derivados de la ciencia y la tecnología.

Dimensión Política: Planear es establecer un compromiso con el futuro, para que una planeación sea variable, debe ubicarse en un marco jurídico institucional que la respalda, aunque en ocasiones es necesario promover algún cambio en el marco en que se circunscribe la planeación.

Dimensión cultural: Cultura entendida como un contexto, un marco de referencia, un sujeto de identidad o una alternativa en el sistema de valores, está siempre presente en toda actividad humana, por tanto, la planeación educativa es afectada por la cultura.

Dimensión prospectiva: Esta es una de las dimensiones de mayor importancia en la planeación, pues al incidir en el futuro hace posible proponer planteamientos inéditos o nuevas realidades. [16] 
Planificar supone un proceso mucho más amplio, que abarca no solo los conceptos didácticos o metodológicos del proceso de enseñanza aprendizaje, sino que incluye todo el conjunto de acciones y decisiones presentes en el contexto escolar. Por eso debemos entender la planificación como una continua y secuencial toma de decisiones basada en el resultado de un sistemático proceso de análisis de la realidad en la que se está operando [17]

La mayoría de la Instituciones Educativas están considerando la planificación curricular integradora con ayuda de aplicativos o sistemas digitales que permite ver la integración, consistencia y coherencia de las categorías didácticas hacia los que se quieren enseñar de manera contextualizada a los intereses de los estudiantes, la realidad social, cultura, política ambiental, etc. a través de las situaciones significativa, este aplicativo integra lo que van a aprender en los estudiantes, como lo van a aprender, con que van aprender y ver como saben que han aprendido y para que les sirven lo que han aprendido. La planificación curricular articulada permitirá alinear estos elementos a fin de tener una mejor planificación que les asegure lograr una ejecución de la sesión de aprendizaje mediados por las herramientas tecnológicas para optimizar sus estrategias.

Las instituciones educativas y centros de investigación han reconocido la importancia de los sistemas de software para el desarrollo óptimo de sus funciones, específicamente en el ámbito de la educación superior el diseño curricular implica un trabajo complejo que requiere entre otras cosas, la administración, coordinación, evaluación y sistematización de sus procesos, ante esto, se requiere del diseño de software que contribuya a mejorar dichos procesos a partir de una cultura de mejora constante. Sin embargo, para proporcionar dicho software se requiere inicialmente de una mejora sustancial en los diferentes planos del proceso de diseño curricular.

El diseño curricular tiene un papel importante respecto a la educación en la sociedad y es necesario considerar el plan de estudios en todas sus etapas como un proceso [18]

El Currículo Nacional en el Perú está integrado por competencias, capacidades y desempeños que ha de ser gestionados con los procesos de enseñanza y aprendizaje a fin de que logren las competencia, como también están sus orientaciones metodologías y de evaluación poniendo énfasis a una de las políticas educativas que es el logros de competencias digitales tanto para el docente y estudiante, que le permitirá desarrollar mejor su enseñanza y el estudiante su aprendizaje e con un sentido más de autonomía, y autoaprendizaje.

La Planificación tiene como propósito fundamental garantizar la cobertura curricular y preparar la enseñanza en correspondencia con las especificaciones curriculares y necesidades que emergerán durante el proceso de enseñanza y aprendizaje (de ahí la relación entre planificación didáctica y planificación curricular a la que antes se hacía referencia), mientras que, por otra parte, el Diseño es la forma particular como cada docente desde sus propias concepciones genera estrategias para llevar a la práctica lo planificado [19]

\section{La Mediación Didáctica En Los Espacios De Formación Virtual}

En los espacios virtuales de enseñanza y aprendizaje, la mediación adquiere una particular importancia en virtud de que la relación entre el docente, el sujeto que conoce y el contenido disciplinar está mediada por las tecnologías. En tal sentido, se asume un enfoque de la didáctica que considera la intervención docente como mediadora entre el sujeto que construye y el objeto de conocimiento.

Este proceso de mediación, independientemente de la modalidad en que se administre, comporta una serie de acciones relacionadas con el diagnóstico del contexto en el cual se lleva a cabo, la toma de decisiones, el desarrollo del proceso de enseñanza y aprendizaje y la evaluación de los resultados de la práctica pedagógica con el objeto de potenciarla y/o reconducirla, si es el caso. Como todo proceso de mediación didáctica complejo e intencional, es difícil preverlo en toda su magnitud, pero es precisamente su complejidad lo que hace necesario tomar previsiones sobre los elementos que lo conforman y la finalidad que se quiere lograr de manera reflexiva, por ello el docente deja de ser un consumidor de prescripciones u orientaciones ajenas y pasa a ser "prosumidor", diseñador y gestor de su trabajo docente [19]

Para la mediación didáctica en los espacios de formación virtual, es necesario partir de la realidad del contexto a través del planteamiento de situaciones significativas que le permita construir problemas del contexto con el uso de las tecnologías acercar la realidad través de estos software virtuales para provocar la mediación entre docente y estudiantes dotándole de consignar ,retroalimentación profunda reflexión a resolver los problemas generando soluciones con el uso de la valoración y sensibilidad pro social.

\section{Aprendizaje significativo utilizando los entornos virtuales en las áreas de comunicación y matemáticas}

Se piensa que el aprendizaje significativo se puede considerar que se ha logrado un aprendizaje cuando el alumno le puede atribuir al contenido un significado y esto solamente sucede si el alumno consigue establecer relaciones sustantivas y no arbitrarias entre el nuevo material de aprendizaje y sus conocimientos previos. Para que un aprendizaje sea significativo debe cubrir dos condiciones: el contenido debe ser potencialmente significativo tanto a nivel lógico como psicológico y el alumno debe presentar una disposición favorable. [20]

La mejora de la enseñanza de las matemáticas pasa por subrayar la importancia de tres aspectos:

- El potencial matemático de las tareas que los profesores proporcionan a sus Estudiantes.

- Las características de la interacción en el aula.

- La manera en la que el profesor propicia el surgimiento

de procesos relevantes de comunicación matemática en el aula [21]

El diseño del software educativo permite construir un conjunto de procesos cognitivos de menor a mayor jerarquía que le permita activar teorías, principios, leyes conceptos para solucionar problemas en el campo de la matemática, comunicación y otras áreas, utilizando procesos como la 
atención, percepción, memoria, razonamiento etc. y aún más relacionar los conocimientos previos con los conocimientos nuevos.

\section{Entornos Virtuales En La Formación Docente}

Entornos virtuales de aprendizaje (EVAs) o en ingles Virtual learning environment (VLE), también conocido por las siglas LMS (Learning Management System), es un espacio alojado en la web, un conjunto de herramientas informáticas que posibilitan la interacción didáctica de manera que el alumno pueda llevar a cabo las labores propias de la docencia como son conversar, leer documentos, realizar ejercicios, formular preguntas al docente, trabajar en equipo, etc. Todo ello de forma simulada sin que medie una interacción física entre docentes y alumnos.

La UNESCO (1998) en su informe mundial de la educación, señala que los entornos de aprendizaje virtuales constituyen una forma totalmente nueva de Tecnología Educativa y ofrece una compleja serie de oportunidades y tareas a las instituciones de enseñanza de todo el mundo, el entorno de aprendizaje virtual lo define como un programa informático interactivo de carácter pedagógico que posee una capacidad de comunicación integrada, es decir, que está asociado a Nuevas Tecnologías. (22)

La educación actual demanda a los futuros docentes o docentes en ejercicio adaptarse a los nuevos tiempos y ampliar las competencias para convertirte en un docente del siglo XXI. Los avances tecnológicos cada vez tienen más influencia en el mundo de la educación facilita un mayor acceso a toda clase de información, así como la introducción de herramientas digitales en el día a día de las clases está prácticamente generalizándose; por lo que se requiere implementar el currículo de formación docente en manejo de entornos virtuales en la formación inicial de docentes y promover especialización para docentes en servicio para que estos mejoren la eficacia y calidad de enseñanza aprendizaje.

Limitar la experiencia educacional al aula es una desventaja para los estudiantes. Con el fácil acceso a la información que hay en la actualidad se puede enseñar y aprender en cualquier momento y en cualquier lugar. Para ello se han desarrollado herramientas y plataformas específicas que responden a estas necesidades, para poder manejarlos y enseñar a los estudiantes a través de ellos es necesario que los profesores se adapten y aprendan a utilizarlos e incorporarlos en sus clases.

\footnotetext{
Krumsvik (2007) provides a definition of digital competence specifically for

Teachers: "Digital competence is the teacher's ability to use ICT with a good

Pedagogical-didactic ICT understanding and to be aware of how this might

Impact the learning strategies and educational formation of pupils" (p.68). (23)
}

Esto significa que el profesor con una buena comprensión pedagógica y didáctica de las TIC debe tomar decisiones sobre qué tipo de herramienta digital se deben utilizar en las diferentes áreas como son comunicación y matemática en cada situación de enseñanza, cómo deben usarse y por qué. Krumsvik señala que es importante desarrollar este tipo de conciencia durante la formación inicial del profesorado.

\section{Plataformas Virtuales}

Las plataformas virtuales, son programas (softwares) orientados a la Internet, se utilizan para el diseño y desarrollo de cursos o módulos didácticos en la red. Permiten mejorar la comunicación (estudiante-docente; estudiante - estudiante) y desarrollar el aprendizaje individual y colectivo en las diferentes áreas como matemáticas, comunicación, inglés, etc.

Una plataforma e-learning, plataforma educativa web o Entorno Virtual de Enseñanza y Aprendizaje es una aplicación web que integra un conjunto de herramientas para la enseñanza-aprendizaje en línea, permitiendo una enseñanza no presencial (e-learning) y/o una enseñanza mixta (b-learning), donde se combina la enseñanza en Internet con experiencias en la clase presencial (PLS Ramboll 2004; Jenkins, Browne y Walker, 2005).

Una plataforma de enseñanza virtual suele estar constituida por tres elementos funcionales o subsistemas:

- LMS (Learning Management System): es el punto de contacto entre los usuarios de la plataforma (profesores y estudiantes, fundamentalmente). Se encarga, entre otras cosas, de presentar los cursos a los usuarios, del seguimiento de la actividad del alumno, etc.

- LCMS (Learning Content Management System): engloba aspectos directamente relacionados con la gestión de contenidos y la publicación de los mismos. También incluye la herramienta de autor empleada en la generación de los contenidos de los cursos.

- Herramientas de comunicación: puesto que la comunicación entre el profesor y el estudiante pasa a ser virtual, deben proporcionarse los mecanismos necesarios para ello. Dentro de este grupo se incluyen Chat, foros, correo electrónico, intercambio de ficheros, etc.

- Estos elementos dan lugar a una serie de herramientas que toda plataforma en mayor o menor medida posee. (24)

\section{Plataformas más usadas}

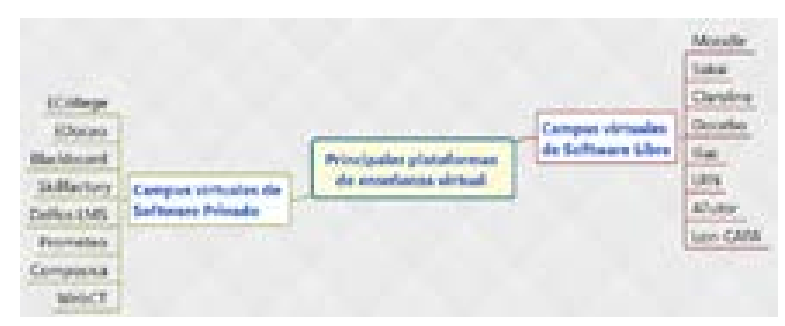

Fig. 4: Principales plataformas de enseñanza

\section{Herramientas Digitales}

Son todos los recursos de software que permite a los docentes la elaboración de sus propios contenidos digitales, estas fomentan la colaboración y facilitar la comunicación entre maestros y estudiantes 
Asi tenemos:

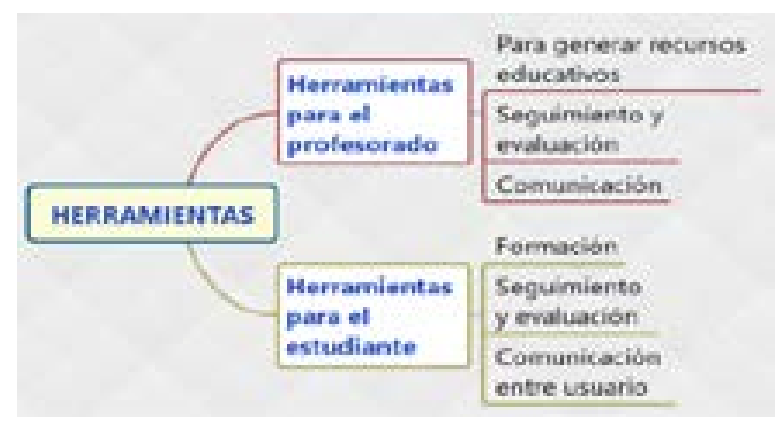

Fig. 5: Herramientas digitales

Algunas herramientas de ayuda

\section{Edmodo}

Edmodo es una herramienta educativa que conecta a profesores y estudiantes, la cual se asimila a una red social. En ésta, los docentes pueden crear grupos colaborativos online, administrar las materias, medir el desempeño de los estudiantes, proveer material académico, comunicarse con los padres de familia, entre otras funciones. Edmodo cuenta con más de 34 millones de usuarios, entre alumnos y docentes, que se conectan para crear un proceso de aprendizaje más enriquecer, personalizado y alineado con las oportunidades que trae la tecnología y el entorno digital.

\section{Socrative}

Diseñado por un grupo de emprendedores e ingenieros apasionados por la educación, Socrative es un sistema que le permite a los profesores realizar ejercicios o juegos educativos, los cuales los estudiantes pueden resolver mediante dispositivos móviles, bien sean smartphones, computadores o tablets. Los maestros pueden ver los resultados de las actividades $\mathrm{y}$, dependiendo de estos, modificar las lecciones posteriores, con el fin de hacerlas más personalizadas.

Projeqted

Projeqted es una herramienta que permite crear presentaciones multimedia, con diapositivas dinámicas en las cuales se pueden incrustar mapas interactivos, links, quizzes online, líneas de tiempo de twitter, videos, entre otras opciones. Durante una sesión de clase, los maestros pueden compartir las presentaciones académicas con sus estudiantes, las cuales se adaptan visualmente a distintos dispositivos.

\section{Thing Link}

Esta herramienta le permite a los educadores crear imágenes interactivas, con música, sonidos, textos, fotografías, entre otras. Éstas pueden ser compartidas en otras páginas web o en redes sociales como Twitter y Facebook. Thing Link brinda la posibilidad de que los maestros creen metodologías de aprendizaje que despierten la curiosidad de los alumnos, por medio de contenidos interactivos que permitan ampliar el conocimiento adquirido.

\section{Scratch}

Scratch es un lenguaje de programación creado por el MIT y especialmente diseñado para que todo el mundo pueda iniciarse en el mundo de la programación. Sirve para crear historias interactivas, juegos y animaciones; además de facilitar la difusión de las creaciones finales con otras personas vía Web.

\section{ClassDojo}

ClassDojo es una herramienta que permite mejorar el comportamiento de los estudiantes: los maestros brindan a sus alumnos retroalimentación instantánea, de tal manera que la buena disposición en clase se 'premia' con puntos, con el fin de que los alumnos tengan una actitud más receptiva frente a su proceso de aprendizaje. Class Dojo provee notificaciones en tiempo real a los estudiantes, como ¡Bien Hecho David! +1 por trabajar colaborativamente. La información que se recolecta sobre el comportamiento de los estudiantes puede ser compartida posteriormente con padres y administradores, todo a través de la Web.

\section{Story Bird}

Story Bird tiene como finalidad fomentar las habilidades de escritura y de lectura en los alumnos, a través del storytelling. En esta herramienta, los maestros pueden crear libros interactivos y artísticos en la Web, por medio de una interfaz sencilla y fácil de usar. Las historias creadas pueden ser incrustadas en blogs, enviadas por correo electrónico, imprimirse, entre otras opciones. En Story Bird, los docentes también pueden crear proyectos con los estudiantes, dar retroalimentación constante, organizar clases y grados, entre otros.

\section{Animoto}

Animoto es una herramienta digital que permite crear videos de alta calidad en poco tiempo y desde cualquier dispositivo móvil, que inspiren a los estudiantes y ayuden a mejorar las lecciones académicas. La interfaz de Animoto es amigable y práctica permitiéndole a los docentes crear contenido audiovisual que se adapte a las necesidades educativas.

\section{XMind}

Xmind es un programa open source para realizar mapas conceptuales, desarrollado por XMind Ltd. Ayuda a la gente a anotar ideas, organizar diversos gráficos, y compartirlos para colaborar online. Soporta mapas mentales, diagramas fishbone, diagramas de árbol, charts organizacionales, charts lógicos, e incluso hojas de cálculo. Usualmente utilizado para organizar conocimientos, tareas.

\section{EdiLIM}

EdiLIM es un entorno para la creación de materiales educativos en formato de Libro Interactivo Multimedia, es compatible con los recursos creados con las versiones anteriores. Los recursos ya creados pueden abrirse con EdiLIM y exportarse al nuevo formato

\section{Kahoot}

Kahoot es una plataforma educativa que se basa en juegos y en preguntas. Por medio de esta herramienta, los profesores pueden crear cuestionarios, discusiones o encuestas que complementen las lecciones académicas, que se proyectan en el aula de clase y que son contestadas por los estudiantes, mientras juegan y aprenden al mismo tiempo. Kahoot permite fomentar una metodología de aprendizaje basada en el juego, que logra incrementar el compromiso de los estudiantes y crear un ambiente educativo dinámico, social y divertido.

\section{Quizizz}

Quizizz es un sitio de web y una herramienta tecnológica gratis que permite a los maestros crear evaluaciones formativas en forma de pruebas pequeñas dentro y afuera de su clase. Esta herramienta conduce los repasos rápidos, ayuda al maestro monitorizar el progreso de un estudiante o una clase. 


\section{Videos en los intercambios de experiencias pasantías}

Aprovechando esta gran ventaja, podemos implementar el uso de videos interactivos, para dos propósitos: en primer lugar, para desarrollar contenidos a través de medios audiovisuales que sirvan como material didáctico y para proponer contenido temático para las clases, en segundo lugar, para evaluar el aprendizaje individual de los alumnos y que permita una retroalimentación puntual y oportuna a cada estudiante.

Es posible llevar a cabo esta estrategia de apoyo al proceso enseñanza-aprendizaje, por medio de programas y aplicaciones computacionales para grabar los videos propios con el contenido y narración de la clase. La plataforma educativa EDpuzzle, permite convertir el video (grabado previamente) en un recurso audiovisual interactivo que el profesor tiene la posibilidad de editar y agregar preguntas (abiertas y de opción múltiple) a lo largo del mismo, así como incluir comentarios y notas de voz, para enfatizar determinados conceptos o contenidos, además puede agregar información adicional que incluya documentos de apoyo, figuras, etc.

Dentro de las innovaciones en los modelos educativos actuales, se encuentra el aprendizaje híbrido y el aula invertida, que emplean videos educativos, uso de simuladores computacionales como laboratorios virtuales de física, desarrollo de actividades experimentales y aplicación de herramientas computacionales para evaluar el aprendizaje y el desarrollo de competencias en los alumnos. Queda claro que ante las nuevas generaciones, se requiere un cambio radical en la forma de llevar a cabo el proceso enseñanzaaprendizaje, o de otra manera habrá serios problemas para que los alumnos adquieran eficientemente el conocimiento y las competencias que demanda el campo laboral actual, es por ello que estrategias de aprendizaje activo e interactivo son de vital importancia para ser aplicadas en este proceso, en particular las estrategias aquí presentadas se adaptan muy bien en los modelos de aula invertida y aprendizaje híbrido.

Es importante mencionar que los videos se utilizaron para lograr la capacitación en forma reflexiva de los docentes, sobre todo observar las experiencias de enseñanza y aprendizaje de como trabajar los entornos virtuales que les permita generar actividades significativas para lograr desarrollar las capacidades de la comprensión lectora y resolución de problemas matemática

\section{MÉTODO}

La investigación tiene como objetivo general fortalecer la formación docente de la mediación de la enseñanza con el uso de los entornos virtuales a través de la implementación del proyecto "Mejoramiento de la calidad del servicio educativo para incrementar el logro de aprendizajes en comunicación y matemática en estudiantes de Instituciones Educativas Públicas del nivel primario, correspondientes al ámbito de intervención de la UGEL Norte" y tiene como objetivos específicos: a) Analizar el manejo de los entornos virtuales como herramientas de medición de la enseñanza, b) Analizar los factores que influyen mejoramiento de su formación profesional con el uso de entornos virtuales .

Selección de los participantes docentes que intervinieron en las siguientes etapas del proyecto "Mejoramiento de la calidad del servicio educativo para incrementar el logro de aprendizajes en comunicación y matemática en estudiantes de Instituciones Educativas Públicas del nivel primario, correspondientes al ámbito de intervención de la UGEL Norte.

\section{Uso de la plataforma}

Se diseñó una plataforma, donde se implementó información sobre planificación curricular y los entornos virtuales que los docentes pudieran recurrir para adquirir conocimiento de acuerdo a sus necesidades, también se solicitó alimentar por cada docente participante con los recurso, herramientas tecnológicas, materiales $\mathrm{u}$ otros insumos que elaboran como material de mediación de la sesión de aprendizaje para realizar un seguimiento y si su elaboración es producto de la capacitación en entorno virtuales.

También se utiliza el Moodle como conexión entre el estudiante y el docente para monitorear y retroalimentar a través de tareas, trabajo de aplicación, actividades etc.

\section{Uso del software educativo}

Los docentes recibieron tres capacitaciones a cargo de expertos de informática para que les enseñara como utilizar las herramientas tecnológicas y que tenían que incluir en la planificación de la programación anual, unidades didácticas y sesiones de aprendizaje es decir en su labor pedagógica

Debiendo utilizar en las ejecuciones de sus sesiones de aprendizaje los espacios virtuales como las aulas de innovación, pizarra digital, cañón multimedia, laptop, xox

\section{Uso de los videos en las pasantías docente}

Se selecciona a algunos docentes que tengan experiencias exitosas para que se les pueda grabar el desarrollo de sus sesiones y luego en reunión masiva se proyecta tres videos para que puedan observar el manejo de los entornos virtuales y puedan hacer un comentario y coevaluación e indicar que si se puede hacer en sus realidades y que compromisos asumieran.

También se complementó con las clases modelos el cual se selecciona a los docentes que necesitan más acompañamiento en el uso de entorno virtuales y el docente experto realiza la clase modelo para que pueda ver aspecto que le son difíciles el manejar y así modelar estrategia que le orienten mejor.

\section{Capacitaciones en la planificación curricular y el} manejo de entorno virtuales

Se realiza tres capacitaciones al año sobre planificación curricular y el uso de los entornos virtuales manejo de los softwares educativos y lo plasman en la planificación de la sesión de aprendizaje utilizando los entornos virtuales

\section{Monitoreo y Acompañamiento}

A cargo de los directores y coordinadores de las TIC, el cual realiza visitas a las aulas de los docentes participantes para observar la utilización de los entornos virtuales, quedando registrado en su ficha de monitoreo, para luego realizar el acompañamiento y asumir mejoras en su trabajo, de igual forma se realiza las estrategias de acompañamiento con el uso de la plataforma, pasantías, clase modelo y las capacitaciones. 


\section{Muestra}

Se seleccionó una muestra al azar de todas las IE de la u gel norte de educación primaria, teniendo una muestra de 84 IE que corresponde a 84 docentes 10 directores

Tabla 3: Ubicación de II.EE. a intervenir. Fuente: Elaboración propia en base a ESCALE

\begin{tabular}{ccc}
\hline $\mathrm{N}^{\circ}$ & DISTRITO & $\begin{array}{c}\text { II.EE. Primaria Poli } \\
\text { docentes }\end{array}$ \\
\hline 1 & Arequipa & 19 \\
2 & Cayma & 22 \\
3 & Cerro Colora-do & 41 \\
\hline Total II.EE. & & \\
\hline
\end{tabular}

\section{Duración}

Se realiza en el periodo 2018

\section{Instrumento}

Se aplicó a los 82 docentes la encuesta de opinión sobre el manejo de entorno virtuales, utilizando la técnica de la encuesta y dos instrumentos una de ellas es el cuestionario que consta de 13 aseveraciones sobre planificación curricular y condiciones de formación docente, con las alternativas de totalmente de acuerdo, de acuerdo, indiferente, en de acuerdo, totalmente de acuerdo, con una valoración de 5 a 1

También se aplicó el otro instrumento cuestionario de 5 preguntas con alternativas de opción múltiple sobre el manejo de los entornos virtuales.

\section{RESULTADOS}

Muestra analizada: Según la muestra de 82 directores y docentes encuestados sobre la formación docente y el manejo de los entornos virtuales como mediación de enseñanza se obtuvo.

Análisis de la fiabilidad: En la dimensión enseñanza que integra 13 ítems, se analizó la fiabilidad del instrumento mediante el método del alfa de cronbach obteniendo una alta fiabilidad, ya que el alfa de cronbach fue de 0.933 .

Tabla 4: Alfa de cronbach de la dimensión enseñanza. Fuente: Encuesta a los 82 docentes la encuesta de opinión sobre el manejo de entorno virtuales

\begin{tabular}{cc}
\hline \multicolumn{2}{c}{ Estadísticos de fiabilidad } \\
\hline Alfa de Cronbach & N de elementos \\
,933 & 13 \\
\hline
\end{tabular}

\section{Dimensiones}

Tabla 5: Promedios de los indicadores de la dimensión enseñanza

\begin{tabular}{|c|c|c|c|c|}
\hline & & $\begin{array}{c}\text { Entorno } \\
\text { virtual }\end{array}$ & Técnica & Dinámica lúdica \\
\hline \multirow{2}{*}{$\mathrm{N}$} & Válidos & 82 & 82 & 82 \\
\hline & Perdidos & 0 & 0 & 0 \\
\hline \multicolumn{2}{|c|}{ Media } & 2.46 & 2.18 & 1.94 \\
\hline \multicolumn{2}{|c|}{ Desv. típ. } & 0.76 & 0.70 & 0.66 \\
\hline
\end{tabular}

Fuente: Encuesta a los 82 docentes la encuesta de opinión sobre el manejo de entorno virtuales. Acerca del entorno virtual el promedio de los 82 encuestados se obtuvo en la puntuación de 2.46 acercándose a la alternativa "de acuerdo" mostrando beneficios al usarlo, también en los 3 ítems que integra tanto planificación virtual y la situación al usarlo, también en los 3 ítems que integra tanto planificación virtual y la situación significativa al estar en el promedio de 2.18 se observa que hay una situación positiva y dicho promedio se acerca a la alternativa "de acuerdo" y con respecto a las actividades lúdicas el promedio de 1.94 ,se interpreta que ha apoyado las lúdicas y estar de
acuerdo.

\section{Efectos del programa}

- P1: En las respuestas de los 82 docentes y directores encuestados 34\%(28 de los 82 evaluados) de ellos afirmo que las estrategias pedagógicas han permitido mejorar los aprendizajes de sus estudiantes.

- P2: La plataforma virtual PIP le ha permitido complementar su labor educativa afirmo 43\%(35 de los 82 evaluados)

- P3. El software que aplican en el área de Matemática, el predominio esta en Scratch con 46\%(lo utilizan 38 de los 82 encuestados)

- P4. El software que se aplica en el área de comunicación es Cmaptool como afirma 34\%(28 de los 82 encuestados) - P5. Acerca de la aplicación del software ha permitido fomentar la creatividad en innovación como afirmaron $38 \%$ de ellos ( 31 de los 82 evaluados)

\section{DISCUSIÓN}

a) En cuanto al análisis del manejo de los entornos virtuales como herramientas de medición de la enseñanza, las estrategias pedagógicas han utilizado como recursos educativos la plataforma virtual PIP, el software, scratch, Cmaptool, contribuyen a mejorar los aprendizajes de sus estudiantes,

fomentado la creatividad e innovación. Por lo tanto, Cabero, J. (2005) corresponde a atender la necesidad de formación y perfeccionamiento del profesorado en TIC, entre ellos destaca fundamentalmente tres:

1.. Es una demanda que no se tiene que ser indiferente la sociedad del conocimiento

2. Las características de los nuevos entornos formativos virtuales en el campo de la educación.

3. Las nuevas formas de enseñar, roles y responsabilidades hacia la modernización de los recursos tecnológicos en el aula

Es decir, la imperiosa necesidad de cambiar las actitudes del profesor ante el uso de las TIC en su quehacer docente.

Es necesario complementarse con la planificación la planificación según Amaro de Chacín, R. (2011). como una continua y secuencial toma de decisiones basada en el resultado de un sistemático proceso de análisis de la realidad en la que se está operando.

Este proceso de mediación, comporta una serie de 
acciones relacionadas con el diagnóstico del contexto en el cual se lleva a cabo, la toma de decisiones, el desarrollo del proceso de enseñanza y aprendizaje y la evaluación de los resultados de la práctica pedagógica con el objeto de potenciarla y/o reconducirla, si es el caso, es así que la mediación juega un papel importante en los procesos de enseñanza del docente para mejorar los logros de aprendizaje de los estudiantes en comunicación y matemática, utilizando los elementos de los entornos virtuales, que ayuden a realizar acciones de razonamiento, resolución ,transferencia, reflexión y creatividad

b) En cuanto al análisis de los factores que influyen mejoramiento de su formación profesional con el uso de entornos virtuales, es la utilización y pertinencia de la capacitación docente, el monitoreo acompañamiento y evaluación de las practicas pedagógicas, la gestión escolar del director, el clima institucional, las condiciones que debe de realizar la gestión directiva que son las condiciones como horario, recursos virtuales, espacios, etc.

\section{CONCLUSIONES}

Las evaluaciones echas al nivel primaria de la región Arequipa correspondiente a la UGEL Norte muestra una brecha significativa por superar estos resultados, por lo que es necesario reorientar la calidad educativa desde la formación inicial docente a través del desarrollo de las competencias digitales que den soporte digital como uso de plataformas, herramientas digitales, entre otros escenarios formativos.

Se demuestra que con la aplicación del programa a través de la encuesta; la mejorar las estrategias de los docentes con el uso de la plataforma virtual PIP, los softwares o herramientas digitales, aunque los resultados muestras que se tiene que seguir implementando en el manejo de estas tecnologías necesarias para mejorar el proceso de enseñanza aprendizaje en las diferentes áreas y niveles.

\section{REFERENCIAS BIBLIOGRÁFICAS}

1. Ministerio de Educacion . Resultados de la evaluacion censal 2007-2014.Recuperado de: http://umc.minedu. gob.pe/informe-de-resultados-de-la-evaluacion-censalde-estudiantes-2007-2015/

2. Organización de las Naciones Unidas(2015).Objetivos del Desarrollo del Milenio Informe 2015.Recuperado de: http://www.un.org/es/millenniumgoals/pdf/2015/ mdg-report-2015_spanish.pdf

3. Ministerio de Educacion(2015).Proyecto educativo nacional al 2021. Lima,Perú Recuperado de : http:// www.minedu.gob.pe/DeInteres/xtras/PEN-2021.pdf

4. Ministerio de Educacion(2012).Marco de buen desempeño docente Lima,Perú..Recuperado de: http://www.minedu.gob.pe/pdf/ed/marco-de-buendesempeno-docente.pdf

5. Minedu (2014).Marco del buen desempeño directivo. Lima,Perú. Recuperado de: http://www.minedu.gob.pe/ DeInteres/xtras/marco_buen_desempeno_directivo.pdf

6. Zapata, M.(2003). Evaluación de un Sistema de Gestión del Aprendizaje.Consultado el 23 de marzo 2008 en :http://www.um.es/ead/aula/calidad/plataformas/eval_ SGA_beta_1.pdf

7. Cabero, J. (2005). "Estrategias para la formación del profesorado en TIC". (Documento en línea):
http://www.pucmm.edu.do/RSTA/Academico/TE/ Documents/fd/efpt.pdf (Consultado el 30 de julio de 2008)

8. Esteve,F. \& Cervera,M(2011).El nuevo paradigma de aprendizaje y las nuevas tecnologias.Recuperado de: https://dialnet.unirioja.es/descarga/articulo/4019253. pdf

9. Alfageme, M.B. (2008). “Análisis del uso de un entorno virtual por el profesorado universitario". Revista Latinoamericana de Tecnología Educativa (RELATEC), vol. $7, \mathrm{n}^{\mathrm{o}} 2$, pp. 17-31. http://campusvirtual.unex.es/ cala/editio/ (Consultado el 12 de diciembre de 2006)

10. Adell, J. (1997): "Tendencias en educación en la sociedad de las Tecnologías de la Información". EDUTEC, Revista Electrónica de Tecnología Educativa, $\mathrm{n}^{\circ} 7$,noviembre de 1997

11. Henríquez, M.A. (2002) "Formación del profesorado en las Tecnologías de la Información y la Comunicación. Casos ULA-URV”. Director, Ángel-Pio González Soto. (Tesis doctoral inédita). Universitat Rovira i Virgili. Facultad de Ciencias de la Educación

12. Bustos Sanchez, A., \& Coll Salvador, C. (2010). LOS ENTORNOS VIRTUALES COMO ESPACIOS DE ENSEÑANZA Y APRENDIZAJE. Una perspectiva psicoeducativa para su caracterización y análisis. Revista Mexicana de Investigación Educativa, 15 (44), 163-184.

13. Infante Moro, A. (Dir.) (2004). "La Enseñanza Virtual en España ante el nuevo Espacio Europeo de Educación Superior. Secretaría de Estado de Universidades e Investigación”. Ministerio de Educación y Ciencia (Proyecto EA2005-0090).

14. CRUE (2006). Las TIC en el Sistema Universitario Español (2006): un análisis estratégico. Madrid:Conferencia de Rectores de las Universidades Españolas (CRUE). (Versión PDF disponible en http:// www.crue.org)

15. Margalef, L. and Álvarez, J. M. (2005). "La formación del profesorado universitario para la innovación en el marco de la integración del Espacio Europeo de Educación Superior". Revista de educación, nº 337, pp. $51-70$

16. Llarena, R., McGinn, N. [et al]. (1981). Definición del campo temario de planeación educativa. En: Documento Base, Congreso Nacional de Investigación Educativa, Vol. I

17. Centro de Experimentación e Investigación Pedagógica del Ministerio de Educación (2012) “Apropiación del currículo con Enfoque en Competencias Laborales en la EMTP". Santiago.Recuperado de : https://educrea. $\mathrm{cl} /$ wp-content/uploads/2016/10/DOC1-planificacion_ didactica.pdf?x16202

18. Panza, 1984

19. Amaro de Chacín, R. (2011). La planificación didáctica $y$ el diseño instruccional en ambientes virtuales. Investigación y Postgrado, 26 (2), 129-160

20. Zuñiga,A.(2002). Aprendizajes significativos en matemáticas quinto grado de educación primaria.(Tesis de Maestro).Recuperado de: https://repositorio.itesm. $\mathrm{mx} /$ bitstream/handle/11285/630177/EGE0000007369. pdf? sequence $=1 \&$ isAllowed $=\mathrm{y}$

21. Llinares, S.; Valls, J. y Roig, A.I. (2008). Aprendizaje 
y diseño de entornos de aprendizaje basado en videos en los programas de formación de profesores de matemáticas. Educación Matemática, 20 (3), pp. 59-82

22. Unesco (1998). La Educación Superior en el Siglo XXI. Visión y Acción. Documento de trabajo de la Conferencia Mundial sobre la Educación Superior. Pag 3 Tomado de: http://investigacion.ilce.edu.mx/panel_ control/doc/c37ambientes.pdf pag 2

23. Ottestad, Geir \& Kelentrić, Marijana \& Guðmundsdóttir, Gréta. (2014). Professional digital competence in teacher education. Nordic Journal of Digital Literacy. P.246

24. Diego Macías Álvarez 2010"Plataformas de enseñanza virtual libres y sus características de extensión: Desarrollo de un bloque para la gestión de tutorías en Moodle" pag. 23-24 